\section{Paying attention to emotion in schizophrenia}

\author{
STEPHAN F. TAYLOR and ISRAEL LIBERZON
}

\begin{abstract}
Schizophrenia represents the pre-eminent 'thought disorder' and a natural subject for a science devoted to analysing human thought. Psychological approaches to the study of schizophrenia seek to describe the mechanisms giving rise to schizophrenic symptoms, and cognitive science has produced a considerable body of experimental data in this area. The psychological construct 'attention' for instance, has motivated and guided useful behavioural studies, such as identifying familial patterns of information-processing, characterising the salutary effects of antipsychotic medication and providing correlations with clinical outcome. However, attention and other notions based on information-processing models do not capture all of schizophrenic phenomenology. Along with a breakdown in associations, Bleuler identified loss of 'affectivity' as one of the primary disturbances of schizophrenia. The negative symptom syndrome - affective flattening, alogia, apathy, amotivation, anhedonia and asociality suggests mental models encompassing more than the processing of pure information, namely, those including emotion.
\end{abstract}

\section{EMOTIONS, SALIENCE AND SCHIZOPHRENIA}

What do we mean by 'emotion'? Emotions permit an organism to attach differential value to stimuli, establish priorities and determine behaviour. As we speak of paying attention to something, we acknowledge that our nervous systems value salient stimuli, such as potential rewards or environmental threats. This selection occurs in regular patterns, which were first placed in an evolutionary context by Charles Darwin. He noted that "serviceable associated habits" evolved to handle common, recurring situations, for example, physical threats leading to fear or domestic threats leading to jealousy. While contemporary students of emotion debate whether cogni- tive appraisal precedes or follows an emotional reaction, normal human mental function is nearly impossible without emotions. Damasio and colleagues have recently argued that emotions allow us to evaluate options, a process occurring prior to conscious awareness of advantageous choices (Bechara et al, 1994). This concept of emotions emphasises their role in setting behavioural priorities, from modulating interest to expressing fear or anger. When one considers the negative symptom syndrome of schizophrenia, in which patients show pervasive deficits of affective drive and modulation, the study of emotion appears very appropriate.

The rediscovery of the negative symptoms of schizophrenia has helped to direct attention to the study of emotional dysregulation in schizophrenia. Several groups have demonstrated that patients with schizophrenia fail to recognise facial affect in others (Walker et al, 1980), with some suggestion that this deficit is not part of generalised cognitive impairment (Gessler et al, 1989). However, other investigators have found that compared with non-emotional perception tasks, patients with schizophrenia did not exhibit greater impairment of affect recognition (Kerr \& Neale, 1993). The reduced range of facial expression remains a core feature of the negative symptom syndrome, but straightforward conclusions about underlying emotional processes are difficult to extract from the data. Systematic assessment, such as facial electromyography, can provide relatively objective descriptions of flattened affect in subjects with schizophrenia. However, in response to emotional probes, patients with flattened affect report no difference in subjective emotional experience (Kring et al, 1993). Clearly, we need a better understanding of the underlying physiology which mediates responses to salient stimuli.

Psychophysiological studies already have a long history in schizophrenia, which can provide useful starting points. For in- stance, numerous studies have found that subjects with schizophrenia exhibit a failure of the normal skin conductance orienting response (reviewed by Bernstein, 1987). Measurement of skin conductance changes has provided an important index of tonic and phasic changes in arousal, providing an essential component of the psychophysiology of emotional response. The wellknown 'polygraph' or 'lie detector' examination utilises this index. The orienting response, which includes phasic increases in skin conductance, signals the presence of salient stimuli. Fittingly then, abnormalities of this system in patients with schizophrenia correlate with the presence of negative symptoms (Bernstein, 1987). Other work shows that in addition to reduced phasic responses of skin conductance to orienting stimuli, patients with schizophrenia exhibit a tonic overactivity of skin conductance, which might reflect ongoing, trait phenomena (Dawson et al, 1994). In response to emotionally-potent film clips, Kring \& Neale (1996) have demonstrated increased skin conductance responses in subjects with schizophrenia. This peripheral measure reflects dysregulation of responses, and not always the absence of response to salient stimuli.

\section{NEUROBIOLOGICAL INSIGHT INTO EMOTION}

Although useful in providing an objective correlate of emotion, skin conductance remains a peripheral measure of central nervous system physiology. The central control of skin conductance depends upon autonomic nuclei in the midbrain and brainstem which regulate sympathetic tone to the peripheral vasculature, but these brain stem regions in turn receive influence from limbic brain, including the ventromedial prefrontal cortex and the amygdaloid complex. In this manner, the peripheral phenomena lead us to the central anatomy and a consideration of its role in schizophrenic phenomenology.

Recent advances in the neurobiological study of emotion permit an investigation of the central systems which generate emotional responses, and limbic and ventromedial frontal regions emerge as major components. Animal models demonstrate the way in which the amygdaloid complex mediates fear conditioning and active avoidance behaviour (Ledoux, 1992). With functional neuroimaging, a new window on 
the in vivo human brain allows one to map emotional processes to specific anatomical structures. This has been demonstrated by mood induction paradigms, which implicate the rostral extent of the medial frontal cortex, including the anterior cingulate cortex, during self-induced dysphoric mood (George et al, 1995). Exposure to aversive visual stimuli leads to activation of the amygdaloid complex (Taylor et al, 1998). The use of pharmacological probes, such as procaine, can stimulate robust cerebral blood flow in medial temporal cortex, including the amygdaloid complex and ventromedial frontal cortex (Ketter et al, 1996). Specific receptor ligands can map the distribution of opioid and neuropeptide systems which concentrate in limbic brain, such as [C-11] carfentanil which identifies dense opioid receptor sites in amygdala, ventromedial/orbital frontal cortex, hypothalamus and thalamus. Such probes will allow the eventual identification of the neurochemistry involved in the modulation of activity in the neuroanatomical circuits described above. While much work lies ahead in characterising the precise roles of brain structures in emotion, the tools to map emotional functions to the limbic anatomy, first proposed by Papez 60 years ago, now exist.

The prefrontal cortex, although more commonly associated with cognitive functions such as working memory, plays a central role in the selection of socially and emotionally appropriate behaviour. We have cited work implicating the ventromedial prefrontal cortex in self-induced dysphoria and skin conductance responses. Another line of evidence comes from neuropsychological studies of frontal lobe lesions, which demonstrate profound effects on personality, that is, an individual's consistent repertoire of emotional responses. Lesions of the ventromedial cortex are associated with an acquired sociopathy, and damage to the lateral prefrontal cortex can cause a psychomotor poverty syndrome. In these cases, individuals exhibit notable deficits in their evaluation of situations, particularly social ones. In the context of emotion, the prefrontal cortex appears to regulate or store the repertoire of behavioural responses that allows an individual to make appropriate judgments and to formulate behaviour in complex social situations. While much work remains in dissecting functional components of the prefrontal cortex, evidence suggests that executive or supervisory functions of the pre- frontal cortex include the regulation of behavioural salience, that is, emotion.

\section{DOPAMINE DYSREGULATION AND SCHIZOPHRENIC SYMPTOMS}

The negative symptoms of schizophrenia may provide a prototypical example of abnormalities of the systems which determine salience. The syndrome spans a wide range of behaviour, from affective modulation of facial expression and speech to motivation and hedonic capacity. Thus, it is hardly surprising that theories of frontal lobe dysfunction should arise to explain schizophrenia. A number of groups have reported correlations between negative symptoms and reduced activity in the lateral prefrontal cortex (Liddle et al, 1992). In the ventromedial cortex, including the adjacent, rostral anterior cingulate cortex, several groups have noted reduced metabolism or blood flow (Andreasen et al, 1992). However, the rather straightforward idea of 'hypofrontality' has not been consistently confirmed. In part, this situation reflects the difficulty of assessing frontal lobe function in an unconstrained, 'rest' state. With activation paradigms, deficient prefrontal lobe function has been a consistent finding, and we would suggest that activation paradigms which evaluate anatomical regions responsible for response priorities and the determination of stimulus salience may provide a fruitful alternative experimental strategy.

Dopaminergic neurons have evolved to signal salience and shape behaviour according to environmental contingencies. These neurons project to prefrontal cortex, particularly medial aspects (Lewis et al, 1987), and modulate activity in response to salient stimuli of diverse types - novel, rewarding, possibly even noxious (reviewed by Salamone et al, 1997). Although dopamine blockers form the mainstay of pharmacotherapy in schizophrenia, little is known about how $\mathrm{D}_{2}$ blockade reduces psychotic symptoms. The relative ineffectiveness of these therapies on negative symptoms has been long recognised, and akinetic sideeffects mimic the negative symptoms of flattened affect and impoverished will. Recent reports of reduced levels of $D_{3}$ and $\mathrm{D}_{4}$ mRNA in the ventromedial frontal cortex (Meador-Woodruff et al, 1997) in subjects with schizophrenia lend support to theories which attribute negative symptoms to reduced dopamine activity in the prefrontal cortex (Weinberger, 1987).

Alternatively, the well-known hypothesis of (striatal) dopaminergic excess in schizophrenia can be formulated as inappropriate attribution of salience, which describes many schizophrenic delusions. Preliminary work showing increased haemodynamic responses of limbic brain to threatening stimuli in patients with schizophrenia of the paranoid subtype has appeared (Phillips et al, 1998). During amphetamine challenge, patients with schizophrenia tend to displace $\mathrm{D}_{2}$ radioligands more than control subjects, consistent with greater release of dopamine (Laruelle et al, 1996). In normal subjects, a complex behavioural task (a video game) appears capable of also causing displacement of the $\mathrm{D}_{2}$ radioligand (Koepp et al, 1998). What remains to be seen is whether exposure to salient stimuli would cause greater release of dopamine in schizophrenia for patients with significant delusions. As we stated above, the autonomic psychophysiology of schizophrenia reveals both excessive and deficient responses. With tools to study in vivo neuroanatomy and neurochemistry, this heterogeneity, so common to any research into mechanisms of psychiatric illness, may yet yield clues about the dysregulation of dopamine in the generation of schizophrenic symptoms.

\section{THE SALIENCE OF EMOTIONS}

The psychological construct of emotion has strong face validity with respect to symptoms of schizophrenia and it can provide an important conceptual linkage to the neuroanatomy and neurochemistry of the disorder. As an organising theme for research into pathophysiology, it has not generated the same level of interest that cognitive psychological constructs, such as attention, have generated. However, in the clinical setting, attentional deficits rarely confront the clinician as deficits in emotion do. An emerging neurobiology of emotion, made possible by recent technological innovations such as functional neuroimaging, suggests that greater attention to emotion research in schizophrenia is now appropriate. If we are ultimately to link schizophrenic phenomena to predisposing genes, associated protein products and pathophysiological processes, we will need to work with relevant phenomenology. Given the multiplicity of psychological constructs, 
many succumb to the temptation to ignore this level of analysis. We argue that emotion, which reflects evolutionary processes embodied in the neural structures which control behaviour, presents a logical, fruitful starting point for this analysis of schizophrenia.

\section{ACKNOWLEDGMENTS}

This work was supported by grants from the National Alliance on Research in Schizophrenia and Depression and the National Institute of Mental Health (K08 MHO1258) to S.F.T.We thank R. Tandon and $E$. Stumbo for their comments on the manuscript.

\section{REFERENCES}

Andreasen, N. C., Rezai, K., Alliger, R., et af (1992) Hypofrontality in neuroleptic-naive patients and in patients with chronic schizophrenia. Archives of General Psychiotry. 49, 943-958.

Bechara, A., Damasio, A. R., Damasio, H., et of (1994) Insensitivity to future consequences following damage to human prefrontal cortex. Cognition, 50, 7-15.

Bernstein, A. S. (1987) Orienting response research in schizophrenia: Where we have come and where we might go, Schizophrenio Bulletin, 13, 623-641.

Dawson, M. E., Nuechterlein, K. H., Schell, A. M., et al (1994) Autonomic abnormalities in schizophrenia. Archives of General Psychiotry, 51, 813-824.

George, M. S., Ketter, T. A., Parekh, P. I., et ol (1995) Brain activity during transient sadness and happiness in healthy women. Americon journal of Psychiatry, 152. $341-351$.

Gessler, S., Cutting, J., Frith, C. D., et ol (1989) Schizophrenic inability to judge facial emotion: a controlled study. British journal of Clinical Psychology. 28. 19-29.

STEPHAN F. TAYLR, MD, Department of Psychiatry, University of Michigan, USA: ISRAEL. LBBERZON, MD. Psychiatry, Veterans Administration Medical Center. Ann Arbor, Michigan, USA

Correspondence: Stephan F. Taylor, Department of Psychiatry. University of Michigan Medical Center. UH 9D Box 0118, I500 East Medical Center Drive. Ann Arbor. MI 48109-0118.USA. Tel: 734-936-9760; Fax: 734-936-4401; e-mail: sftaylor Qumich.edu

(First received 26 March 1998, final revision 24 August 1998, accepted 26 August 1998)

Kerr, S. L. \& Neale, J. M. (1993) Emotion perception in schizophrenia: specific deficit or further evidence of generalized poor performance? Journal of Abnormal Psychology, 102, 312-318

Ketter, T. A., Andreason, P. J., George, M. S., et ol (1996) Anterior paralimbic mediation of procaineinduced emotional and psychosensory experiences. Archives of General Psychiatry, 53, 59-69.

Koepp, M. J., Gunn, R. N., Lawrence, A. D., et al (1998) Evidence for striatal dopamine release during a video garne. Noture, 393, 266-26.

Kring, A. M., Kerr, S. L., Smith, D. A., et al (1993) Flat affect in schizophrenia does not reflect diminished subjective experience of emotion. Journal of Abnormal Psychology. 102, 507-517.

_ Neale, J.M. (1996) Do schizophrenic patients show a disjunctive relationship among expressive. experiential, and psychophysiological components of emotion? Journal of Abnormal Psychology. 105, 249-257.

Laruelle, M., Abi, D. A., van Dyck, C. H., et al (1996) Single photon emission computerized tomography imaging of amphetamine-induced dopamine release in drug-free schizophrenic subjects. Proceedings of the Notional Acodemy of Sciences of the United States of America, 93. 9235-9240

Ledoux, J. E. (1992) Emotion and the amygdala. In The Amygdala: Neurobiological Aspects of Emotion. Memory and Mental Dysfunction (ed J. P. Aggleton). pp 339-351. New York: Wiley-Liss.

Lewis, D. A., Foote, S. L., Goldstein, M., et al (1987) The distribution of tyrosine hydroxylase- immunoreactive fibers in primate neocortex is widespread but regional specific. Journal of Neuroscience. 7. $279-290$.

Liddle, P. F., Friston, K. J., Frith, C. D., et al (1992) Patterns of cerebral blood flow in schizophrenia. British journal of Psychiatry, 160, 179-186.

Meador-Woodruff, J. H., Haroutunian, V., Powchik, P., et al (1997) Dopamine receptor transcript expression in striatum and prefrontal and occipital cortex. Archives of General Psychiotry, 54. 1089-1095.

Phillips, M. L., Young, A. W., Senior, C., et al (1998) Specific neural substrates for disgust and fear in normal and clinical groups. Biological Psychiotry, 43, 735.

Salamone, J. D., Cousins, M. S. \& Smyder, B. .) (1997) Behavioral functions of nucleus accumbens dopamine: empirical and conceptual problems with the anhedonia hypothesis. Neuroscience and Biobehovioral Reviews, 21. 341-359.

Taylor, S. F., Liberzon, L., Fig, L. M., et al (1998) The effect of emotional content on visual recognition memory: A PET activation study. Neurolmage, in press.

Walker, E., Marwit, S. J. \& Emory, E. (1980) A crosssectional study of emotion recognition in schizophrenics. journal of Abnormal Psychology, 89, 428-436.

Weinberger, D. R. (1987) Implications of normal brain development for the pathogenesis of schizophrenia. Archives of General Psychiatry, 44, 660-669. 